\title{
TIME-HARMONIC ACOUSTIC SCATTERING FROM LOCALLY PERTURBED HALF-PLANES*
}

\author{
GANG $\mathrm{BAO}^{\dagger}$, GUANGHUI HU ${ }^{\ddagger}$, AND TAO YIN $§$
}

\begin{abstract}
This paper is concerned with time-harmonic acoustic scattering of plane waves in one or two inhomogeneous half-planes with an unbounded interface. The contrast function is supposed to have a compact support, while the infinite interface is a local perturbation of the $x_{1}$-axis. For an acoustically impenetrable interface, the scattering phenomenon occurs in one half-plane only and the impedance (Robin) boundary value problem is investigated. In the penetrable case, we study a transmission problem in two half-planes. Our approach for forward scattering is based on the finite element method in a truncated bounded domain coupled with the boundary element method. Numerical experiments are tested to verify our scheme. For the inverse problem, we prove that the near-field data of a finite number of incoming plane waves or a single point source wave uniquely determine the shape of a rectangular cavity of impedance-type.
\end{abstract}

Key words. acoustic scattering, locally perturbed half-planes, coupled method, inverse scattering, uniqueness

AMS subject classifications. 35C15, 65N21, 65N30, 65N38

DOI. $10.1137 / 18 \mathrm{M} 1164068$

1. Introduction. The scattering problems in a locally perturbed half-space in $\mathbb{R}^{n}(n=2,3)$ have attracted much attention over the last 20 years. They have many applications in remote sensing, ocean acoustics, geophysics, outdoor sound propagation, and so on. Throughout this paper, the concept local perturbation means a one-dimensional (1D) curve which coincides with the $x_{1}$-axis in $|x|>R$ for some $R>0$. This type of interface is not necessarily the graph of a compactly supported function over the $x_{1}$-axis. In this paper, we assume that a time-harmonic plane wave is incident onto a locally perturbed interface separating two isotropic media in two dimensions and investigate both the direct and inverse scattering problems.

In the time-harmonic regime, it is well known that the total field can be decomposed into three parts: the incoming wave $u^{i n}$, the reflected wave $u^{r e}$ corresponding to the unperturbed scattering interface (i.e., the straight line $x_{2}=0$ in $2 \mathrm{D}$ ), and the scattered wave $u^{s c}$ caused by the presence of local perturbations. Under the Sommerfeld radiation condition of $u^{s c}$ in the half-space, one can show uniqueness and the existence of weak solutions if the total field fulfills a Dirichlet or Neumann boundary condition, whereas the reflected waves are usually uniquely determined by Snell's law in physics; see, e.g., $[1,2,3,33,24,26,27,32,36]$.

\footnotetext{
*Received by the editors January 5, 2018; accepted for publication (in revised form) August 1, 2018; published electronically October 16, 2018.

http://www.siam.org/journals/siap/78-5/M116406.html

Funding: The first author's work was supported in part by an NSFC Innovative Group Fund (11621101), an Integrated Project of the Major Research Plan of NSFC (91630309), and an NSFC A3 Project (11421110002). The second author's work was supported by the NSFC grant (11671028) and NSAF grant (U1530401). The third author's work was partially supported by the NSFC grant (11501063).

${ }^{\dagger}$ School of Mathematical Sciences, Zhejiang University, Hangzhou 310027, China (baog@zju. edu.cn).

¥Beijing Computational Science Research Center, Beijing 100193, China (hu@csrc.ac.cn).

$\S$ Department of Computing \& Mathematical Sciences, California Institute of Technology, 1200 E. California Blvd., Pasadena, CA 91125 (taoyin89@caltech.edu).
} 
Due to the local perturbation of the infinite interface, variational and integral equation methods (see, e.g., $[1,2,27,36,33]$ ) can be adopted to reduce the unbounded physical domain to a truncated computational domain. This has significantly simplified the calculation and analysis over an unbounded domain for treating general rough surface scattering problems [11, 7, 9, 20, 22, 35, 32]. However, these approaches rely heavily on the reflection principle of the Helmholtz equation under a Dirichlet or Neumann boundary condition and can hardly be extended to the Robin case. One reason lies in the fact that the reflection principle with the Robin boundary condition is not of the point-to-point type; see, e.g., [19, 23]. In fact, a transparent boundary condition (or nonreflecting boundary operator) always requires a series expansion of half-plane Sommerfeld radiation solutions with the corresponding boundary condition on $x_{2}=0$. However, unlike the Dirichlet and Neumann cases [27, 33], it is not trivial to derive such an expansion for the Robin boundary problem. On the other hand, to the best of our knowledge, it remains unclear how to apply the integral equation approach, with the free-space fundamental solution being the kernel (see [36] in the Dirichlet case), to a bounded computational domain for treating the Robin boundary value problem in a locally perturbed half-space. The authors in [12] studied an equivalent integral system involving the impedance Green's function in the half-space but did not provide a proof of the existence of solutions.

The aim of this paper is twofold. First, we shall review and remark on solvability results for Dirichlet and Neumann boundary value problems; see section 2.1. Our new contribution is to mathematically justify the reflected waves in appropriate Sobolev spaces, leading to new insights into the Dirichlet, Neumann, and Robin boundary value problems under question. Especially in the Neumann case, we shall explain why surface waves are excluded for local perturbations. Further, using a coupling scheme between the boundary integral equation and variational methods, we prove the well-posedness of the scattering problems with the Robin boundary condition and transmission interface conditions; see sections 2.2 and 2.3. Our coupling scheme is closest to the lines of [21]. Compared to [26,3], we avoid the calculations of hypersingular integral operators. The proposed approach seems promising since it can be used to handle various boundary value problems in a locally perturbed halfspace, provided that the Green's function to the unperturbed problem fulfills the Sommerfeld radiation condition. The solvability result for transmission problems fills the gap in [15]; see Remark 2.3. Numerical tests will be reported in section 4 .

Second, we shall consider the inverse problem of determining the perturbed scattering interface from near-field measurement data $[4,5,6]$. We prove that the nearfield data of a finite number of incoming plane waves (with distinct incident directions or wavenumbers) or a single point source wave uniquely determine the shape of a rectangular cavity of impedance type.

\section{Forward scattering problems.}

2.1. Half-plane scattering problems for impenetrable interfaces. Assume that a time-harmonic incoming wave $u^{i n}$ is incident onto the straight line $\Gamma_{0}:=\left\{x_{2}=0\right\}$ from the upper half-plane $\mathbb{R}_{+}^{2}:=\left\{\left(x_{1}, x_{2}\right): x_{2}>0\right\}$. The propagation of the incident wave in an isotropic homogeneous medium can be modeled by the Helmholtz equation

$$
\Delta u^{i n}+k^{2} u^{i n}=0 \quad \text { in } \quad \mathbb{R}^{2}
$$

with the wavenumber $k>0$. In this paper, $u^{i n}$ is assumed to be a plane wave of the form 


$$
u^{i n}=u^{i n}(x ; d)=\exp (i k x \cdot d)=\exp \left(i k\left(x_{1} \sin \theta-x_{2} \cos \theta\right)\right),
$$

where $d=(\sin \theta,-\cos \theta) \in \mathbb{S}:=\{x:|x|=1\}$ stands for the incident direction and $\theta \in(-\pi / 2, \pi / 2)$ the incident angle. Suppose that the total wave field $u=u^{i n}+u^{r e}$ does not penetrate into the lower half-space $\mathbb{R}_{-}^{2}:=\left\{\left(x_{1}, x_{2}\right): x_{2}<0\right\}$, where $u^{\text {re }}$ denotes the outgoing reflected wave scattered back from $\Gamma_{0}$. We consider one of the following three boundary conditions imposed on $\Gamma_{0}$ :

$$
\begin{array}{ll}
\text { Dirichlet boundary condition: } & u=0 ; \\
\text { Neumann boundary condition: } & \partial_{\nu} u=0 ; \\
\text { Robin boundary condition: } & \partial_{\nu} u+i k \lambda u=0,
\end{array}
$$

where $\nu \in \mathbb{S}$ is the unit normal at $\Gamma_{0}$ directed into $\mathbb{R}_{+}^{2}$ (that is, $\nu=(0,1)$ ) and $\lambda \in \mathbb{C}$ satisfies $\operatorname{Re} \lambda>0, \operatorname{Im} \lambda \geq 0$. In $x_{2}>0$, we suppose that the outgoing wave $u^{r e}$ satisfies the upward angular spectrum representation (UASR) proposed in [11, 9]:

$$
u^{r e}(x)=\frac{1}{\sqrt{2 \pi}} \int_{\mathbb{R}} \exp \left(i\left[\left(x_{2}-h\right) \sqrt{k^{2}-\xi^{2}}+x_{1} \xi\right]\right) \hat{u}_{h}^{r e}(\xi) d \xi, \quad x_{2}>h,
$$

for any $h>0$. Here $\sqrt{k^{2}-\xi^{2}}=i \sqrt{\xi^{2}-k^{2}}$ when $\xi^{2}>k^{2}$, where $\hat{u}_{h}^{r e}(\xi)$ denotes the Fourier transform of $u^{r e}\left(x_{1}, h\right)$ with respect to $x_{1}$, i.e.,

$$
\hat{u}_{h}^{r e}(\xi)=\mathcal{F}_{x_{1} \rightarrow \xi}\left[u^{r e}\left(x_{1}, h\right)\right]:=\frac{1}{\sqrt{2 \pi}} \int_{\mathbb{R}} \exp \left(-i x_{1} \xi\right) u^{r e}\left(x_{1}, h\right) d x_{1}, \quad \xi \in \mathbb{R} .
$$

In fact, for $u^{r e}\left(x_{1}, h\right) \in L^{2}(\mathbb{R})$, the UASR condition (2.4) can be written in the form

$$
u^{r e}(x)=2 \int_{\mathbb{R}} \frac{\partial \Phi(x, y)}{\partial y_{2}} u^{r e}\left(y_{1}, h\right) d y_{1}, \quad x_{2}>h,
$$

which is called the upward propagating radiation condition (UPRC) (see [13, 14]). Here $\Phi(x, y)$ is the free-space fundamental solution to the Helmholtz equation in $\mathbb{R}^{2}$ given in (2.12).

The UASR can be derived for bounded outgoing waves in general settings. The representation (2.4) shows that $u^{r e}$ is a linear superposition of the upward propagating plane waves $\exp \left(i x_{1} \xi+i x_{2} \sqrt{k^{2}-\xi^{2}}\right)$ for $|\xi| \leq k$ and evanescent surface waves $\exp \left(i x_{1} \xi+i x_{2} \sqrt{k^{2}-\xi^{2}}\right)$ for $|\xi|>k$. Hence, the radiation condition UASP covers plane waves, surface waves, and the Sommerfeld outgoing waves which propagate into the upper half-plane. We refer to [13, Theorem 2.9 (ii)] for the proof that the Sommerfeld radiation condition is stronger than the UPRC. For $h>0$, define the strip between $\Gamma_{0}$ and $\Gamma_{h}$ by $\widetilde{U}_{h}:=\left\{x: 0<x_{2}<h\right\}$. For $s, \varrho \in \mathbb{R}$, introduce the weighted Sobolev spaces

$$
\widetilde{V}_{h, \varrho}:=\left\{u:\left[\int_{\widetilde{U}_{h}}\left(\left|\left(1+\left|x_{1}\right|^{2}\right)^{\varrho / 2} u\right|^{2}+\left|\nabla\left[\left(1+\left|x_{1}\right|^{2}\right)^{\varrho / 2} u\right]\right|^{2}\right) d x\right]^{1 / 2}<\infty\right\}
$$

and

$$
H_{\varrho}^{s}(\mathbb{R}):=\left(1+x_{1}^{2}\right)^{-\varrho / 2} H^{s}(\mathbb{R}),
$$

where $H^{s}(\mathbb{R})$ is identified with the Sobolev space $H^{s}(\mathbb{R})$ with norm

$$
\|v\|_{H^{s}(\mathbb{R})}=\left(\int_{\mathbb{R}}\left(1+\xi^{2}\right)^{s}|\mathcal{F} v|^{2} d \xi\right)^{1 / 2}
$$

Copyright $\odot$ by SIAM. Unauthorized reproduction of this article is prohibited. 
The weighted space $H_{\varrho}^{s}(\mathbb{R})$ will be endowed with the norm

$$
\|v\|_{H_{\varrho}^{s}(\mathbb{R})}:=\left\|\left(1+x_{1}^{2}\right)^{\varrho / 2} v\left(x_{1}\right)\right\|_{H^{s}(\mathbb{R})} .
$$

Obviously, the restriction of the incident plane wave $u^{i n}$ given in $(2.2)$ to $\widetilde{U}_{h}$ belongs to the space $\widetilde{V}_{h, \varrho}$ for all $\varrho<-1 / 2$ and $h>0$. Moreover, given $u^{r e} \in \widetilde{V}_{h, \varrho}$ for $\varrho>-1$, it holds that $\left.u^{r e}\right|_{\Gamma_{h}} \in H_{\varrho}^{1 / 2}(\mathbb{R})$ and $\hat{u}_{h}^{r e} \in H_{\varrho}^{1 / 2}(\mathbb{R})$, and that the integral (2.4) can be interpreted as a linear functional between $H_{\varrho}^{1 / 2}(\mathbb{R})$ and its dual space $H_{-\varrho}^{-1 / 2}(\mathbb{R})$; see [9]. Below we present solvability results of the boundary value problems (2.3) and (2.4), which will be referred to as unperturbed scattering problems.

\section{THEOREM 2.1.}

(i) In the Dirichlet or impedance case, there exists a unique solution to the halfplane scattering problem in the weighted Sobolev space $\widetilde{V}_{h, \varrho}$ with $h>0$ and $\varrho \in(-1,-1 / 2)$. Moreover, the solution $u^{\text {re }}$ is of the form

$$
u^{r e}(x)=-\exp \left(i k\left(x_{1} \sin \theta+x_{2} \cos \theta\right)\right)
$$

in the Dirichlet case and

$$
u^{r e}(x)=\frac{\cos \theta-\lambda}{\cos \theta+\lambda} \exp \left(i k\left(x_{1} \sin \theta+x_{2} \cos \theta\right)\right)
$$

in the impedance case.

(ii) Under the Neumann boundary condition, all solutions $u^{r e} \in \widetilde{V}_{h, \varrho}$ with $h>0$ and $\varrho \in(-1,-1 / 2)$ can be expressed as

$$
\begin{aligned}
u^{r e}(x)= & \exp \left(i k\left(x_{1} \sin \theta+x_{2} \cos \theta\right)\right) \\
& +C^{+} \exp \left(i k x_{1}\right)+C^{-} \exp \left(-i k x_{1}\right)
\end{aligned}
$$

for some $C^{ \pm} \in \mathbb{C}$.

Proof. The case of the Dirichlet or Robin boundary value problem follows from the solvability results for unbounded rough surface scattering problems in $\mathbb{R}^{2}$, where the scattering interface is allowed to be a global perturbation of the $x_{1}$-axis. We refer to $[9,22,20]$ for the details.

In the Neumann case, it is easy to verify that $u^{r e}=\exp \left(i k\left(x_{1} \sin \theta+x_{2} \cos \theta\right)\right) \in$ $\widetilde{V}_{h, \varrho}$ is indeed a solution. Let $w$ be another solution and set $v:=u^{r e}-w$. Then $v$ satisfies the UASR

$$
v(x)=\frac{1}{\sqrt{2 \pi}} \int_{\mathbb{R}} \exp \left(i x_{2} \sqrt{k^{2}-\xi^{2}}+i x_{1} \xi\right) \hat{v}(\xi, 0) d \xi, \quad x_{2}>0,
$$

together with the homogeneous boundary condition $\partial_{2} v=0$ on $x_{2}=0$. Note that $\partial_{2} v\left(x_{1}, 0\right) \in H_{\varrho}^{-1 / 2}(\mathbb{R})$, since $v \in \widetilde{V}_{h, \varrho}$ for any $h>0$ and $\varrho \in(-1,-1 / 2)$ by our assumption. It is easy to observe that

$$
0=\mathcal{F}_{x_{1} \rightarrow \xi}\left[\partial_{2} v\left(x_{1}, 0\right)\right]=i \sqrt{k^{2}-\xi^{2}} \hat{v}(\xi, 0) \in H_{\varrho}^{-1 / 2}(\mathbb{R}) .
$$

Since $H_{\varrho}^{-1 / 2}(\mathbb{R})$ is the dual space of $H_{-\varrho}^{1 / 2}(\mathbb{R})$, the above relation is understood as

$$
\int_{\mathbb{R}} \sqrt{k^{2}-\xi^{2}} \hat{v}(\xi, 0) f(\xi) d \xi=0 \quad \text { for all } \quad f \in H_{1 / 2}^{s}(\mathbb{R})
$$

Copyright (c) by SIAM. Unauthorized reproduction of this article is prohibited. 
with $s \in(1 / 2,1)$. In particular, the identity (2.8) holds for all smooth functions with compact support. This implies that $\sqrt{k^{2}-\xi^{2}} \hat{v}(\xi, 0)=0$ in the distributional sense. Hence, $\hat{v}(\xi, 0)=C^{+} \delta(\xi-k)+C^{-} \delta(\xi+k)$ for some $C^{ \pm} \in \mathbb{C}$. Inserting the expression of $\hat{v}(\xi, 0)$ into $(2.7)$ yields

$$
\begin{aligned}
v(x) & =C^{+} \mathcal{F}_{\xi \rightarrow k}^{-1}\left[e^{i x_{2} \sqrt{k^{2}-\xi^{2}}} \delta(\xi-k)\right]+C^{-} \mathcal{F}_{\xi \rightarrow k}^{-1}\left[e^{i x_{2} \sqrt{k^{2}-\xi^{2}}} \delta(\xi+k)\right] \\
& =C^{+} \exp \left(i k x_{1}\right)+C^{-} \exp \left(-i k x_{1}\right),
\end{aligned}
$$

which belongs to $\widetilde{V}_{h, \varrho}$ for all $\rho \in(-1 / 2,-1)$ and $h>0$. The proof of the second assertion is complete.

The expressions for $u^{r e}$ in Theorem 2.1 (choose $C^{+}=C^{-}=0$ in the Neumann case) can be physically interpreted by Snell's law. From the second assertion, one can conclude that uniqueness to the Neumann problem does not hold in general in the weighted Sobolev space $\widetilde{V}_{h, \varrho}$ with $\rho \in(-1 / 2,-1)$. Since the UASR covers surface waves (which exponentially decays as $x_{2} \rightarrow+\infty$ ), the second assertion shows that surface waves do not exist in the half-plane scattering problem under the Neumann boundary condition. Note that the right-hand side of (2.6) contains no exponentially decay terms.

Next we introduce the half-plane Sommerfeld radiation condition, which ensures the uniqueness of the Neumann boundary value problem in a locally perturbed halfplane.

DeFInITION 2.2. The function $v$ is said to satisfy the half-plane Sommerfeld radiation condition if the relation

$$
\lim _{r \rightarrow \infty} \sqrt{r}\left(\partial_{r} v-i k v\right)=0, \quad r=|x|, \quad x \in\{|x|>R\} \cap \mathbb{R}_{+}^{2}
$$

holds uniformly in all directions $\hat{x} \in \mathbb{S}^{+}$.

Note that the half-plane Sommerfeld radiation condition is stronger than UASR. That is, if $v$ satisfies (2.9), then $v$ must fulfill the UASR, but not vice versa. The above radiation condition implies the asymptotic behavior in the upper half-plane

$$
v(x)=\frac{e^{i k r}}{\sqrt{r}}\left(v^{\infty}(\hat{x})+O\left(\frac{1}{r}\right)\right), \quad r=|x| \rightarrow \infty,
$$

uniformly in all directions $\hat{x} \in \mathbb{S}^{+}$, where $v^{\infty}$ is called the far-field pattern of the radiation solution $v$.

In a vast literature (see, e.g., [33, 1, 27]), it has been proved that the perturbed acoustic scattering problems (see section 2.2 below for the formulation) with the Dirichlet and Neumann boundary conditions admit a unique solution $u \in H_{l o c}^{1}\left(\Omega^{+}\right)$ of the form

$$
\begin{gathered}
u=u^{i n}+u^{r e}+u^{s c} \\
u^{r e}:= \begin{cases}-\exp \left(i k\left(x_{1} \sin \theta+x_{2} \cos \theta\right)\right) & \text { in the Dirichlet case } \\
\exp \left(i k\left(x_{1} \sin \theta+x_{2} \cos \theta\right)\right) & \text { in the Neumann case }\end{cases}
\end{gathered}
$$

where $u^{s c}=u-u^{i n}-u^{r e}$ satisfies the half-plane Sommerfeld radiation condition. Evidently, the assumption on $u^{s c}$ gives rise to $C^{ \pm}=0$ in the Neumann case (see the second assertion of Theorem 2.1), since $\exp \left( \pm i k x_{1}\right)$ does not fulfill (2.9). This explains why uniqueness of the Neumann boundary value problem could be proved with 


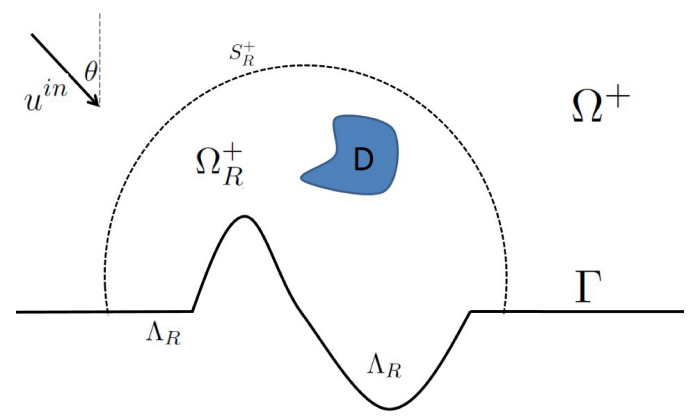

FIG. 1. Geometrical settings for the impedance boundary value problem. A plane wave is incident from above and $\Lambda_{R}=\Gamma \cap\{|x|<R\}$ is the locally perturbed part.

the Sommerfeld radiation condition for $u^{s c}$. However, one can construct nontrivial solutions to the homogeneous Neumann boundary value problem (i.e., $u^{i n}=0$ ) that fulfill the UASR other than (2.9); see Remark 2.2 below.

The remaining part of this section is devoted to well-posedness of forward scattering problems with the Robin boundary condition and transmission conditions.

2.2. Scattering from locally perturbed half-planes: Impedance case. Assume a time-harmonic plane wave $u^{i n}(x ; d)$, with $d \in \mathbb{S}$ being the incident direction, is incident onto a bounded penetrable scatterer $D$ embedded in a homogeneous isotropic half-plane $\Omega^{+}$. The boundary $\Gamma:=\partial \Omega^{+} \subset \mathbb{R}^{2}$ is assumed to be a Lipschitz continuous curve that coincides with the straight line $\Gamma_{0}:=\left\{x_{2}=0\right\}$ in $\left|x_{1}\right|>R$ for some $R>0$; see Figure 1. We emphasize that the perturbed part, which we denote by $\Lambda_{R}:=\Gamma \cap\left\{x:\left|x_{1}\right|<R\right\}$, is not necessarily the graph of some function. We refer to them as scattering problems from locally perturbed half-planes, due to the presence of the bounded scatterer $D \subset \mathbb{R}^{2}$ and the curved surface $\Lambda_{R}$. The acoustic property of the background medium in $\Omega^{+}$can be characterized by the refractive function $q \in L^{\infty}\left(\Omega^{+}\right)$such that $q \equiv 1$ in $\Omega^{+} \backslash \bar{D}$ (after some normalization). Equivalently, the contrast function $1-q$ is compactly supported in $D$. In this section, we suppose that the total field $u$ satisfies the Robin boundary condition on $\Gamma$. The wave propagation of the total field is then governed by the following boundary value problem for the Helmholtz equation

$$
\Delta u+k^{2} q u=0 \quad \text { in } \quad \Omega^{+}, \quad \partial_{\nu} u+i k \lambda u=0 \quad \text { on } \quad \Gamma,
$$

where $\nu \in \mathbb{S}$ is the unit normal at $\Gamma$ directed into $\Omega^{+}$. Let $a>0$ be such that $D \subset\left\{x: x_{2}<a\right\}$ and let $V_{h, \varrho}$ be defined as the same as $\tilde{V}_{h, \varrho}$ with $\Gamma_{0}$ replaced by $\Gamma$. Denote by $u^{r e}$ the outgoing reflected field corresponding to the unperturbed problem (i.e., $D=\emptyset$ and $\Gamma=\Gamma_{0}$ ); see Theorem 2.1. Below we state well-posedness of the forward scattering problem with local perturbations.

Theorem 2.3. Assume that $\lambda \in \mathbb{C}$ satisfies $\operatorname{Re} \lambda>0, \operatorname{Im} \lambda \geq 0$. Then, for any incident plane wave at the wavenumber $k>0$, there exists a unique solution $u \in H_{\text {loc }}^{1}\left(\Omega^{+}\right)$of the form $u=u^{i n}+u^{r e}+u^{s c}$ for (2.11), where the scattered field $u^{s c}$ fulfills the half-space Sommerfeld radiation condition (2.9). Moreover, if $\Lambda_{R}$ is the graph of some Lipschitz function, then $u$ coincides with the unique solution in the weighted Sobolev space $V_{h, \varrho}$ for all $h>a$ and $\varrho \in(-1,-1 / 2)$ such that $u-u^{\text {in }}$ satisfies the UASR (2.4). 
If $\Lambda_{R}$ (and thus $\Gamma$ ) is the graph of some Lipschitz function, then the existence and uniqueness of $u-u^{i n}$ in $V_{h, \varrho}$ follow from the well-posedness of rough surface scattering problems with generalized impedance boundary conditions [22]. The above theorem implies that the unique solution $u-u^{i n} \in V_{h, \varrho}$ satisfying the UASR consists of an outgoing plane wave $u^{r e}$ and a Sommerfeld radiating solution $u^{s c}$ in the upper half-space.

It suffices to prove the first part of Theorem 2.3. For this purpose, we need the free-space fundamental solution $\Phi(x, y)$ to the Helmholtz equation $\left(\Delta+k^{2}\right) u=0$ in $\mathbb{R}^{2}$, given by

$$
\Phi(x, y)=\frac{i}{4} H_{0}^{(1)}(k|x-y|), \quad x, y \in \mathbb{R}^{2}, x \neq y .
$$

Here $H_{0}^{(1)}$ is the Hankel function of the first kind of order zero. Denote by $G(x, y)$ $\left(y \in \mathbb{R}_{+}^{2}\right)$ the Green's function to the unperturbed impedance boundary value problem, i.e.,

$$
\begin{aligned}
\left(\Delta+k^{2}\right) G(x, y) & =-\delta(x-y) & & \text { in } \quad \mathbb{R}_{+}^{2}, \\
\left(\partial_{2}+i k \lambda\right) G(\cdot, y) & =0 & & \text { on } \quad x_{2}=0 .
\end{aligned}
$$

Evidently, $G(x, y)$ can be regarded as the total field incited by the point source wave $u^{i n}=G(\cdot, y)$ incident on $\Gamma_{0}$. Below we present the expression of $G$ and its far-field behavior (see, e.g., [10]).

Lemma 2.4. Green's function $G(\cdot, y)\left(y \in \mathbb{R}_{+}^{2}\right)$ can be expressed as

$$
\begin{aligned}
& G(x, y)=\Phi(x, y)+\Phi\left(x, y^{\prime}\right)-P(x, y), \\
& P(x, y):=\frac{i \lambda}{2 \pi} \int_{\mathbb{R}} \frac{e^{i k\left[\left(y_{1}+y_{2}\right)\left(1-s^{2}\right)^{1 / 2}-\left(x_{1}-x_{2}\right) s\right]}}{\left(1-s^{2}\right)^{1 / 2}\left[\left(1-s^{2}\right)^{1 / 2}+\lambda\right]} d s
\end{aligned}
$$

with $\operatorname{Im} \sqrt{\cdot} \geq 0$. Here $y^{\prime}=\left(y_{1},-y_{2}\right)$ for $y=\left(y_{1}, y_{2}\right) \in \mathbb{R}^{2}$. Moreover, the function $G(\cdot, y)$ satisfies the half-space radiation condition (2.9), with the far-field pattern given by

$$
G^{\infty}(\hat{x}, y)=\exp (-i k \hat{x} \cdot y)+\frac{\cos \theta-\lambda}{\cos \theta+\lambda} \exp \left(-i k \hat{x} \cdot y^{\prime}\right)
$$

where $\hat{x} \in \mathbb{S}^{+}:=\{(\cos \theta, \sin \theta): \theta \in(0, \pi)\}$.

If $\lambda=0$, then $P(x, y)=0$ and $G(x, y)$ coincides with the Green's function satisfying the Neumann condition on $\Gamma_{0}$, which can be easily obtained by the method of images. The correction term $P(x, y)$ given by $(2.13)$ can be derived via Fourier transform. We refer to $[10,7,8]$ for an asymptotic expansion of $P$ as $|x| \rightarrow \infty$ and alternative representations of $P$ in the form of Laplace-type integrals which are suitable for numerical evaluation. The proof of Lemma 2.4 can be found in [7, Chapter 2.1]. It is easy to observe that the far-field pattern of $G$ fulfills the relation $G^{\infty}(\hat{x}, y)=u^{i n}(y ;-\hat{x})+u^{r e}(y ;-\hat{x})$, where $u^{i n}(x ; d)$ and $u^{r e}(x ; d)$ are given by $(2.2)$ and (2.5), respectively.

Our proof of Theorem 2.3 is based on the variational argument in a truncated bounded domain $\Omega_{R}^{+}:=\left\{x \in \Omega^{+}:|x|<R\right\}$ coupled with a Dirichlet-to-Neumann map derived from the integral representation of $u^{s c}$ in $\left\{x \in \mathbb{R}^{+}:|x|>R\right\}$. Similar coupling schemes were used in $[26,3]$ for treating the transmission problem with a locally perturbed medium and a flat interface (i.e., $D \neq \emptyset$ and $\Gamma=\left\{x_{2}=0\right\}$ ) as well as the Dirichlet and Neumann boundary value problems. 
A variational formulation coupled with an integral equation is derived as follows. Let $\partial \Omega_{R}^{+}=S_{R}^{+} \cup \Lambda_{R}$ be the boundary of $\Omega_{R}^{+}$, where $S_{R}^{+}:=\left\{x: x \in \Omega^{+},|x|=R\right\}$. Let $\gamma \subset \partial \Omega$ be a subboundary of some boundary domain $\Omega \subset \mathbb{R}^{2}$. Introduce the spaces (see, e.g., [28])

$$
H^{1 / 2}(\gamma):=\left\{\left.u\right|_{\gamma}: u \in H^{1 / 2}(\partial \Omega)\right\}, \quad \tilde{H}^{1 / 2}(\gamma):=\left\{u \in H^{1 / 2}(\partial \Omega): \operatorname{supp}(u) \subset \gamma\right\} .
$$

Then we denote by $H^{-1 / 2}(\gamma)$ the dual space of $\tilde{H}^{1 / 2}(\gamma)$ and by $\tilde{H}^{-1 / 2}(\gamma)$ the dual space of $H^{1 / 2}(\gamma)$. It is easy to derive the following variational formulation for $u \in$ $H^{1}\left(\Omega_{R}^{+}\right)$:

$$
\int_{\Omega_{R}^{+}} \nabla u \cdot \nabla \bar{\varphi}-k^{2} q u \bar{\varphi} d x-\int_{S_{R}^{+}} \partial_{\nu} u \bar{\varphi} d s-i k \lambda \int_{\Lambda_{R}} u \bar{\varphi} d s=0
$$

for all $\varphi \in H^{1}\left(\Omega_{R}^{+}\right)$, where $\nu \in \mathbb{S}$ is the normal on $S_{R}^{+}$pointing into the exterior of $\Omega_{R}^{+}$. Choosing $R_{1}>R$ and applying Green's formula for $u^{s c}$ to the region $\Omega_{R_{1}}^{+} \backslash \overline{\Omega_{R}^{+}}$ yields

$u^{s c}(x)=\left(-\int_{S_{R_{1}}^{+}}+\int_{S_{R}^{+}}\right)\left[u^{s c}(y) \partial_{\nu} G(x ; y)-\partial_{\nu} u^{s c}(y) G(x ; y)\right] d s(y), \quad x \in \Omega_{R_{1}}^{+} \backslash \overline{\Omega_{R}^{+}}$.

Note that both $u^{s c}$ and $G(\cdot, y)$ satisfy the impedance boundary condition on $\Lambda_{R_{1}} \backslash \Lambda_{R}$. Letting $R_{1} \rightarrow \infty$ and making use of the asymptotic behavior of $u^{s c}$ and $G$ as $|x| \rightarrow \infty$ yields

$$
u^{s c}(x)=\int_{S_{R}^{+}}\left[u^{s c}(y) \partial_{\nu} G(x ; y)-\partial_{\nu} u^{s c}(y) G(x ; y)\right] d s(y), \quad x \in \Omega^{+} \backslash \overline{\Omega_{R}^{+}} .
$$

Taking the limit $x \rightarrow S_{R}^{+}$in $(2.15)$ and setting $p:=\left.\partial_{\nu} u^{s c}\right|_{S_{R}^{+}} \in \tilde{H}^{-1 / 2}\left(S_{R}^{+}\right)$, we get

$$
(I-\mathcal{D})\left(\left.u^{s c}\right|_{S_{R}^{+}}\right)+\mathcal{S} p=0 \quad \text { on } \quad S_{R}^{+} .
$$

Here $I$ is the identify operator, $\mathcal{D}$ and $\mathcal{S}$ are the double and single layer operators over $S_{R}^{+}$, respectively, defined by

$$
\begin{aligned}
& (\mathcal{D} g)(x):=2 \int_{S_{R}^{+}} \partial_{\nu(y)} G(x ; y) g(y) d s(y), \quad x \in S_{R}^{+}, \\
& (\mathcal{S} p)(x):=2 \int_{S_{R}^{+}} G(x ; y) p(y) d s(y), \quad x \in S_{R}^{+} .
\end{aligned}
$$

We remark that the jump relations for $\mathcal{D}$ and $\mathcal{S}$ remain valid, since $G(\cdot ; y)-\Phi(\cdot ; y)$ is of $C^{\infty}$-smoothness in $\Omega^{+}$. Combining (2.14) and (2.16) gives the variational formulation for the unknown solution pair $(u, p) \in H^{1}\left(\Omega_{R}^{+}\right) \times \tilde{H}^{-1 / 2}\left(S_{R}^{+}\right)=: X$ as follows:

$$
A((u, p),(\varphi, \chi)):=\left(\begin{array}{l}
a_{1}((u, p),(\varphi, \chi)) \\
a_{2}((u, p),(\varphi, \chi))
\end{array}\right)=\left(\begin{array}{c}
\int_{S_{R}^{+}} \partial_{\nu} v \bar{\varphi} d s \\
\int_{S_{R}^{+}}(I-\mathcal{D})\left(\left.v\right|_{S_{R}^{+}}\right) \bar{\chi} d s
\end{array}\right)
$$

for all $(\varphi, \chi) \in X$, where $v:=u-u^{s c}=u^{i n}+u^{r e}$ and

$$
\begin{aligned}
& a_{1}((u, p),(\varphi, \chi)):=\int_{\Omega_{R}^{+}} \nabla u \cdot \nabla \bar{\varphi}-k^{2} q u \bar{\varphi} d x-\int_{S_{R}^{+}} p \bar{\varphi} d s-i k \lambda \int_{\Lambda_{R}} u \bar{\varphi} d s, \\
& a_{2}((u, p),(\varphi, \chi)):=\int_{S_{R}^{+}}\left[(I-\mathcal{D})\left(\left.u\right|_{S_{R}^{+}}\right)+\mathcal{S} p\right] \bar{\chi} d s .
\end{aligned}
$$

Copyright $@$ by SIAM. Unauthorized reproduction of this article is prohibited. 
Remark 2.1. The variational formulation (2.17) and our scattering problem are equivalent in the following sense. If $u=u_{0}+u^{s c}$ is a solution to our original problem, then the restriction of $u$ to $\Omega_{R}^{+}$satisfies the variational equation (2.17). On the other hand, if $u \in X$ is a solution to (2.17), then solution $u$ can be extended from $\Omega_{R}^{+}$to $\Omega^{+}$via $u=u^{i n}+u^{r e}+u^{s c}$, where $u^{s c}$ is expressed by (2.15) in terms of the trace of $u^{s c}$ on $S_{R}^{+}$. It follows from (2.16) that the jump of $u^{s c}$ is continuous at $S_{R}^{+}$. The jump of $\partial_{\nu} u^{s c}$ is also continuous at $S_{R}^{+}$, since the operator $\mathcal{S}$ is always invertible under the Robin boundary condition on $\Lambda_{R}$. Hence, $u \in H_{l o c}^{1}\left(\Omega^{+}\right)$and $u-u_{0}$ satisfies the radiation solution $(2.9)$.

Below we shall verify uniqueness and existence of solutions to (2.17).

Proof of Theorem 2.3. Assuming $u^{i n}=0$, we have $u=u^{s c}$. Taking the imaginary part of (2.14) with $\varphi=u=u^{s c}$, we see

$$
-k \operatorname{Re}(\lambda) \int_{\Lambda_{R}}\left|u^{s c}\right|^{2} d s=\operatorname{Im}\left(\int_{S_{R}^{+}} \partial_{\nu} u^{s c} \overline{u^{s c}} d s\right) .
$$

Making use of the radiation condition of $u^{s c}$ and the Cauchy-Schwarz inequality, we find

$$
\begin{aligned}
\operatorname{Im}\left(\int_{S_{R}^{+}} \partial_{\nu} u^{s c} \overline{u^{s c}} d s\right) & =\operatorname{Im}\left(\int_{S_{R}^{+}}\left(\partial_{\nu} u^{s c}-i k u^{s c}\right) \overline{u^{s c}} d s\right)+k \int_{S_{R}^{+}}\left|u^{s c}\right|^{2} d s \\
& \rightarrow k \int_{S^{+}}\left|u^{\infty}(\hat{x})\right|^{2} d s
\end{aligned}
$$

as $R \rightarrow \infty$. Hence, the right-hand side of (2.18) is nonnegative for sufficiently large $R>0$. Since $\operatorname{Re}(\lambda)>0$, this implies that $u^{s c}=0$ and thus $\partial_{\nu} u^{s c}=0$ on $\Lambda_{R}$ for large $R>0$. Applying Holmgren's uniqueness theorem (see, e.g., [16, Theorem 2.3]) gives $u^{s c} \equiv 0$ in $\Omega^{+}$. This finishes the proof of uniqueness. To prove existence, we only need to show that the variational formulation (2.17) is of Fredholm type.

Denote by $(\cdot, \cdot)$ the $L^{2}$ duality between $H^{1}\left(\Omega_{R}^{+}\right)$and $H^{-1}\left(\Omega_{R}^{+}\right)$and by $<\cdot, \cdot>$ the $L^{2}$ duality between $H^{1 / 2}\left(S_{R}^{+}\right)$and $\tilde{H}^{-1 / 2}\left(S_{R}^{+}\right)$, respectively. By the Riesz representation theorem, there exist linear operators

$$
\begin{gathered}
T_{1}, J_{1}: \quad H^{1}\left(\Omega^{+}\right) \rightarrow H^{-1}\left(\Omega^{+}\right), \\
T_{2}: \tilde{H}^{-1 / 2}\left(S_{R}^{+}\right) \rightarrow H^{-1}\left(\Omega^{+}\right), \\
T_{3}: \tilde{H}^{-1 / 2}\left(S_{R}^{+}\right) \rightarrow H^{1 / 2}\left(S_{R}^{+}\right), \\
J_{2}: \quad H^{1}\left(\Omega_{R}^{+}\right) \rightarrow H^{1 / 2}\left(S_{R}^{+}\right)
\end{gathered}
$$

such that for $(u, p),(\varphi, \chi) \in X$,

$$
\begin{aligned}
\left(T_{1} u, \varphi\right) & :=\int_{\Omega_{R}^{+}} \nabla u \cdot \nabla \bar{\varphi}+u \bar{\varphi} d x \\
\left(J_{1} u, \varphi\right) & :=-\int_{\Omega_{R}^{+}}\left(k^{2} q-1\right) u \bar{\varphi} d x-i k \lambda \int_{\Lambda_{R}} u \bar{\varphi} d s, \\
\left\langle T_{2} p, \varphi\right\rangle & :=\int_{S_{R}^{+}} p \bar{\varphi} d s \\
\left\langle T_{3} p, \chi\right\rangle & :=\int_{S_{R}^{+}} \mathcal{S} p \bar{\chi} d s \\
\left\langle J_{2} u, \chi\right\rangle & :=-\int_{S_{R}^{+}} \mathcal{D}\left(\left.u\right|_{S_{R}^{+}}\right) \bar{\chi} d s .
\end{aligned}
$$

Copyright (c) by SIAM. Unauthorized reproduction of this article is prohibited. 
By Sobolev embedding theorems, $J_{1}$ is compact. The operator $J_{2}$ is also compact, since the double layer operator $\mathcal{D}: H^{1 / 2}\left(S_{R}^{+}\right) \rightarrow H^{1 / 2}\left(S_{R}^{+}\right)$is compact (see [28]). Then we may rewrite $A: X \times X \rightarrow \mathbb{C}^{2}$ as

$$
A((u, p),(\varphi, \chi))=\left\langle A_{1}(u, p),(\varphi, \chi)\right\rangle+\left\langle A_{2}(u, p),(\varphi, \chi)\right\rangle
$$

where $\langle\cdot, \cdot\rangle$ denotes the duality between $X$ and $X^{\prime}$, and the operators $A_{j}: X \rightarrow X^{\prime}$ $(j=1,2)$ are defined as

$$
A_{1}:=\left(\begin{array}{cc}
T_{1} & -T_{2} \\
T_{2}^{*} & T_{3}
\end{array}\right), \quad A_{2}:=\left(\begin{array}{cc}
J_{1} & 0 \\
J_{2} & 0
\end{array}\right) .
$$

Since $T_{1}$ and $T_{3}$ are coercive operators over $H^{1}\left(\Omega_{R}^{+}\right)$and $\tilde{H}^{-1 / 2}\left(S_{R}^{+}\right)$(see [28]), respectively, the real part of $A_{1}$, given by

$$
\operatorname{Re} A_{1}:=\frac{A_{1}+A_{1}^{*}}{2}=\left(\begin{array}{ll}
T_{1} & \\
& T_{3}
\end{array}\right)
$$

is coercive over $X$. Since $A_{2}$ is compact, the operator $A$ is Fredholm type with index zero. Applying the Fredholm alternative yields the existence of solutions.

Having obtained the trace $u^{s c}, \partial_{\nu} u^{s c}$ on $S_{R}^{+}$from (2.17), we may calculate the far-field data through (cf. (2.15))

$$
u^{\infty}(\hat{x})=\int_{S_{R}^{+}}\left[u^{s c}(y) \partial_{\nu} G^{\infty}(\hat{x} ; y)-\partial_{\nu} u^{s c}(y) G^{\infty}(\hat{x} ; y)\right] d s(y), \quad \hat{x} \in \mathbb{S}^{+},
$$

where $G^{\infty}(\hat{x}, y)$ is given in Lemma 2.4 .

Remark 2.2. If $\lambda=0$, one can construct a nontrivial solution $v$ to the homogeneous Neumann problem (i.e., $u^{i n}=u^{r e} \equiv 0$ ) with local perturbations, which fulfills the UASR. In fact, set $g^{ \pm}(x)=C^{ \pm} \exp \left( \pm i k x_{1}\right)$ for some $C^{ \pm} \in \mathbb{C}$. We claim that we can find a Sommerfeld radiation solution $v^{s c} \in H_{l o c}^{1}\left(\Omega^{+}\right)$such that $v=g^{ \pm}+v^{s c}$ solves the homogeneous boundary value problem

$$
\left(\Delta+k^{2}\right) v=0 \quad \text { in } \quad \Omega^{+}, \quad \partial_{\nu} v=0 \quad \text { on } \quad \Gamma .
$$

If $\Gamma$ coincides with $x_{2}=0$, it holds that $v^{s c}=0$ and thus $v=g^{ \pm}$. In the case of local perturbations, the existence and uniqueness of $v^{s c}$ follow from existing arguments for cavity scattering problems; see $[1,33,27]$. Note that the constructed solution $v$ satisfies the UASR but not the Sommerfeld radiation condition.

2.3. Transmission problems in a two-layered medium. In this subsection, we shall carry over the proposed coupling scheme under the impedance boundary value problem to transmission conditions. Our aim is to derive an equivalent variational formulation in a bounded domain and then apply the Fredholm alternative. The uniqueness of solutions was verified in $[29,25]$ for more general locally perturbed interfaces. We also refer to $[30,34,31]$ for solvability results obtained via the limiting absorption principle which requires an a priori estimate of the solutions.

We preserve the notation used before and introduce new ones as follows. Let $\Omega^{-}$denote the unbounded region below $\Gamma, \Omega_{R}^{-}=\left\{x \in \Omega^{-}:|x|<R\right\}, S_{R}^{-}:=$ $\left\{x \in \Omega^{-}:|x|=R\right\}$. Denote by $q_{-}$the refractive index function characterizing the inhomogeneous medium in $\Omega^{-}$such that $q_{-} \equiv 1$ in $\Omega^{-} \backslash \bar{D}_{-}$, where $D_{-} \subset \Omega^{-}$is a 


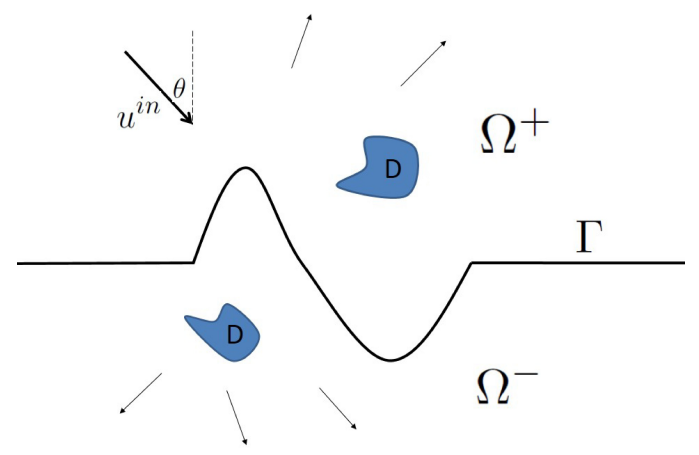

FIG. 2. Geometrical settings for the transmission problem. $D=D^{+} \cup D^{-}$are inhomogeneous media embedded above and below $\Gamma$, respectively.

bounded domain; see Figure 2. If an incident wave from $\Omega^{+}$could penetrate into the $\Omega^{-}$, the total field $u$ can be modeled by the transmission problem

$$
\begin{array}{ll}
\Delta u_{ \pm}+k_{ \pm}^{2} q_{ \pm} u_{ \pm}=0 & \text { in } \Omega^{ \pm}, \\
u_{+}=u_{-}, \quad \partial_{\nu}^{+} u_{+}=\eta \partial_{\nu}^{-} u_{-} & \text {on } \Gamma, \quad \eta \in \mathbb{C},
\end{array}
$$

where $u_{ \pm}=\left.u\right|_{\Omega^{ \pm}}, q_{+}=q$, and $k_{+}=k, k_{-} \in \mathbb{R}$ are the wavenumbers in $\Omega^{ \pm}$, respectively.

Denote by $u_{ \pm}^{r e}$ the upward $(+)$ and downward $(-)$ propagating fields corresponding to the unperturbed scattering problem (that is, $\Gamma=\Gamma_{0}, D=\emptyset, D_{-}=\emptyset$ ), which satisfy

$$
\begin{aligned}
& \Delta u_{ \pm}^{r e}+k_{ \pm}^{2} u_{ \pm}^{r e}=0 \quad \text { in } \quad \mathbb{R}_{ \pm}^{2}:=\left\{x: x_{2} \gtrless 0\right\} \\
& u^{i n}+u_{+}^{r e}=u_{-}^{r e}, \quad \partial_{\nu}^{+}\left[u^{i n}+u_{+}^{r e}\right]=\eta \partial_{\nu}^{-}\left[u_{-}^{r e}\right] \quad \text { on } \quad \Gamma_{0}:=\left\{x: x_{2}=0\right\} .
\end{aligned}
$$

At infinity, we suppose that $u_{ \pm}^{r e}$ satisfy the upward and downward angular spectrum representations. By [22], the solutions $u_{ \pm}^{r e} \in V_{ \pm h, \varrho}$ are uniquely solvable for all $h>a$ and $\varrho \in(-1,-1 / 2)$. Here the number $a>0$ is chosen such that $D^{+} \cup D^{-} \subset\left\{x:\left|x_{2}\right|<\right.$ $a\}$ and the Sobolev space $V_{-h, \varrho}$ is defined in the same way as $\tilde{V}_{h, \varrho}$ (see subsection 2.1) with $\Gamma_{0}$ and $\Gamma_{h}$ replaced by $\Gamma$ and $\Gamma_{-h}$, respectively. If $u^{i n}=e^{i\left(\alpha x_{1}-\beta^{+} x_{2}\right)}$ with $\alpha=k_{+} \cos \theta, \beta^{+}=k_{+} \sin \theta$, then by the Fresnel formula, $u_{ \pm}^{r e} \in V_{ \pm h, \varrho}$ can be expressed as

$$
\begin{array}{rlr}
u_{+}^{r e}(x) & =\frac{\beta^{+}-\eta \beta^{-}}{\beta^{+}+\eta \beta^{-}} e^{i\left(\alpha x_{1}+\beta^{+} x_{2}\right)}, & x_{2}>0, \\
u_{-}^{r e}(x) & =\frac{2 \beta^{+}}{\beta^{+}+\eta \beta^{-}} e^{i\left(\alpha x_{1}-\beta^{-} x_{2}\right)}, & x_{2}<0, \\
\beta^{-} & :=\sqrt{k_{-}^{2}-k_{+}^{2} \cos ^{2} \theta} .
\end{array}
$$

In the transmission case, we look for functions

$$
u_{+}^{s c}:=u_{+}-u^{i n}-u_{+}^{r e} \quad \text { in } \quad \Omega^{+}, \quad u_{-}^{s c}:=u_{-} u_{-}^{r e} \quad \text { in } \Omega^{-},
$$

which fulfill the half-plane radiation condition (2.10) in the upper and lower halfplanes, respectively. 
To derive the variational formulation, we denote again by $G(x, y)$ the Green's function to the unperturbed transmission problem, i.e.,

$$
\begin{aligned}
& \left(\Delta+k_{ \pm}^{2}\right) G(x, y)=-\delta(x-y) \quad \text { in } \quad \mathbb{R}^{2} \backslash\{y\}, y \notin \Gamma, \\
& G^{+}(\cdot, y)=G^{-}(\cdot, y), \quad \partial_{\nu}^{+} G^{+}(\cdot, y)=\eta \partial_{\nu}^{-} G^{-}(\cdot, y) \quad \text { on } \quad x_{2}=0
\end{aligned}
$$

with the Sommerfeld radiation condition (2.10) in each half-space. We refer to [30, $34,31,18]$ for the existence, uniqueness, and representations of $G$.

Let $R>0$ be sufficiently large such that the truncated domain $\Omega_{R}:=\{x$ : $\left.|x|<R, x \in \mathbb{R}^{2}\right\}$ contains the penetrable inhomogeneities $D=D^{+} \cup D^{-}$. Obviously, $\partial \Omega_{R}=S_{R}^{+} \cup \Lambda_{R} \cup S_{R}^{-}$. Analogously to the impedance boundary value problem, we may derive the following variational formulation: Find $u \in H^{1}\left(\Omega_{R}\right)$ and $p=\left(p^{+}, p^{-}\right) \in$ $\tilde{H}^{-1 / 2}\left(S_{R}^{+}\right) \times \tilde{H}^{-1 / 2}\left(S_{R}^{-}\right)$such that

$$
\begin{aligned}
& A((u, p),(\varphi, \chi))=\left(\begin{array}{c}
\int_{S_{R}^{+}} \partial_{\nu} v \bar{\varphi} d s+\eta \int_{S_{R}^{-}} \partial_{\nu} v \bar{\varphi} d s \\
\int_{S_{R}^{+}}\left(I-\mathcal{D}^{+}\right)\left(\left.v\right|_{S_{R}^{+}}\right) \overline{\chi^{+}} d s+\eta \int_{S_{R}^{-}}\left(I-\mathcal{D}^{-}\right)\left(\left.v\right|_{S_{R}^{-}}\right) \overline{\chi^{-}} d s
\end{array}\right), \\
& A((u, p),(\varphi, \chi)):=\left(\begin{array}{l}
a_{1}((u, p),(\varphi, \chi)) \\
a_{2}((u, p),(\varphi, \chi))
\end{array}\right)
\end{aligned}
$$

for all $\varphi \in H^{1}\left(\Omega_{R}\right)$ and $\chi=\left(\chi^{+}, \chi^{-}\right) \in \tilde{H}^{-1 / 2}\left(S_{R}^{+}\right) \times \tilde{H}^{-1 / 2}\left(S_{R}^{-}\right)$. Here

$$
\begin{aligned}
\left.v\right|_{\Omega^{ \pm}}=u-u_{ \pm}^{s c}=u^{i n}+u_{ \pm}^{r e} & \\
a_{1}((u, p),(\varphi, \chi)):= & \int_{\Omega_{R}^{+}} \nabla u_{+} \cdot \nabla \bar{\varphi}-k_{+}^{2} q_{+} u_{+} \bar{\varphi} d x-\int_{S_{R}^{+}} p^{+} \bar{\varphi} d s \\
& +\eta\left\{\int_{\Omega_{R}^{-}} \nabla u_{-} \cdot \nabla \bar{\varphi}-k_{-}^{2} q_{-} u_{-} \bar{\varphi} d x-\int_{S_{R}^{-}} p^{-\bar{\varphi}} d s\right\}, \\
a_{2}((u, p),(\varphi, \chi)):= & \int_{S_{R}^{+}}\left[\left(I-\mathcal{D}^{+}\right)\left(\left.u\right|_{S_{R}^{+}}\right)+\mathcal{S}^{+} p^{+}\right] \overline{\chi^{+}} d s \\
& +\eta\left\{\int_{S_{R}^{-}}\left[\left(I-\mathcal{D}^{-}\right)\left(\left.u\right|_{S_{R}^{-}}\right)+\mathcal{S}^{-} p^{-}\right] \overline{\chi^{-}} d s\right\} .
\end{aligned}
$$

The single and double layer operators are defined via

$$
\begin{array}{ll}
\left(\mathcal{D}^{ \pm} g\right)(x):=2 \int_{S_{R}^{ \pm}} \partial_{\nu(y)} G(x ; y) g(y) d s(y), & x \in S_{R}^{ \pm}, \\
\left(\mathcal{S}^{ \pm} f\right)(x):=2 \int_{S_{R}^{ \pm}} G(x ; y) f(y) d s(y), & x \in S_{R}^{ \pm} .
\end{array}
$$

By arguing analogously to the proof of Theorem 2.17, one can prove that the operator $A$ satisfies Gardinger's inequality over $X:=H^{1}\left(\Omega_{R}\right) \times \tilde{H}^{-1 / 2}\left(S_{R}^{+}\right) \times \tilde{H}^{-1 / 2}\left(S_{R}^{-}\right)$. We omit the details, since the proofs are quite similar. Hence, by the Fredholm alternative we obtain the following.

THEOREM 2.5. For any incident plane wave at the wavenumber $k>0$, there exists a unique solution $(u, p) \in X$ to the variational formulation (2.22). Hence, the transmission problem (2.19) and (2.21) admit a unique solution $u=u^{i n}+u^{r e}+u^{s c}$.

Remark 2.3. Theorem 2.5 corrects the solvability results presented in [15]. When $\eta=1$ and $D=\emptyset$, the authors there claimed that the unique solution takes the explicit representation (see [15, Theorem 2.2])

Copyright $\odot$ by SIAM. Unauthorized reproduction of this article is prohibited. 


$$
u^{s c}(x)=\int_{\Lambda_{R}}\left[G(x, y) \partial_{\nu} u^{s c}(y)-\partial_{\nu} G(x, y) u^{s c}(y)\right] d s(y) \quad \text { for all } \quad x \in \mathbb{R}^{2} \backslash \Lambda_{R} .
$$

In fact, the above representation holds for $|x|>R$ only, and it does not satisfy the governing equation in the region between $\Lambda_{R}$ and $x_{2}=0$.

3. Inverse scattering from rectangular cavities of impedance type. In this section, we consider the inverse problem of recovering the shape of a rectangular cavity of impedance type. Assume that the scattering interface $\Gamma$ coincides with $x_{2}=0$ in $|x|>R$ for some $R>0$ and the perturbed section $\Lambda_{R} \subset \Gamma$ consists of line segments parallel to $x_{1^{-}}$or $x_{2}$-axis only. Such a geometry models a rectangular cavity above or below the ground plane $x_{2}=0$. Further, we suppose that the region $\Omega^{+}$ above $\Gamma$ consists of homogeneous media and satisfies the geometrical assumption

$$
\left(x_{1}, x_{2}\right) \in \Omega^{+} \rightarrow\left(x_{1}, x_{2}+s\right) \in \Omega^{+} \text {for all } s>0 .
$$

Let $u^{\text {in }}$ be either an incoming plane wave of the form (2.2) or a point source wave with the source position located at $y \in \Omega_{R}^{+}$(see (2.12)). We assume that the impedance coefficient $\lambda$ is given as a priori data and the near-field data are measured on the line segment

$$
I=\left\{\left(x_{1}, h\right):\left|x_{1}\right|<C\right\} \text { for some } C>0, h>R .
$$

The following theorem asserts that the data of a finite number of incoming plane waves or one point source wave can be used to uniquely determine $\Lambda_{R}$. Moreover, we show that the number of incoming waves required depends on the height of the cavity. The deeper the cavity, the more the incoming plane waves. This kind of uniqueness result is similar to that given by Colton and Sleeman [17] for inverse scattering from a bounded sound-soft obstacle. It was proved in [17] that the shape of a sound-soft obstacle can be uniquely determined by the far-field pattern of a finite number of incident plane waves provided a priori information on the size of the obstacle is available. We remark that the idea of Colton and Sleeman cannot apply to a bounded obstacle with a Neumann or Robin boundary condition, due to the lack of monotonicity of the eigenvalues of the negative Laplacian for these boundary conditions; see [16, Chapter 5]. Our arguments show that, at least for rectangular cavities, one can get uniqueness with a finite number of incoming plane waves.

THEOREM 3.1.

(i) Suppose that there is a priori information that $\max \left\{\left|x_{2}\right|:\left(x_{1}, x_{2}\right) \in \Gamma\right\}<H$ and let $M$ be the largest integer less than $2 H k / \pi$. Then $\Lambda_{R}$ can be uniquely determined by the near-field data set $I$ of $2 M+1$ plane waves with distinct incident directions $d_{j}=\left(\sin \theta_{j},-\cos \theta_{j}\right)$ with $\cos \theta_{j} \neq \lambda, j=1,2, \ldots, 2 M+1$, and one fixed wavenumber.

(ii) Let $H$ be given as in case (i) and assume $k_{j} \leq K$ for some upper bound $K \in \mathbb{R}^{+}, j=1,2, \ldots ; N+1$, where $N$ as the largest integer less than $2 H K / \pi$. Then $\Lambda_{R}$ can be uniquely determined by the near-field data set I of incoming plane waves with one fixed direction and $N+1$ wavenumbers.

(iii) Let $u^{i}$ be a point source wave. Then the near-field data measured on I uniquely determine $\Lambda_{R}$.

Proof. Let $\Gamma^{(1)}$ and $\Gamma^{(2)}$ be two rectangular perturbations of $x_{2}=0$. Denote by $u_{j}^{s c}$ and $u_{j}$ the scattered and total fields corresponding to $\Gamma^{(j)}(j=1,2)$ (see Theorem 2.3), and by $\Omega_{j}^{+}$the unbounded domain above $\Gamma^{(j)}$. Assuming $u_{1}=u_{2}$ on $I$, we need to prove that $\Lambda_{R}^{(1)}=\Lambda_{R}^{(2)}$, where $\Lambda_{R}^{(j)}:=\Lambda^{(j)} \cap\{|x|<R\}$ denotes the perturbed part. 


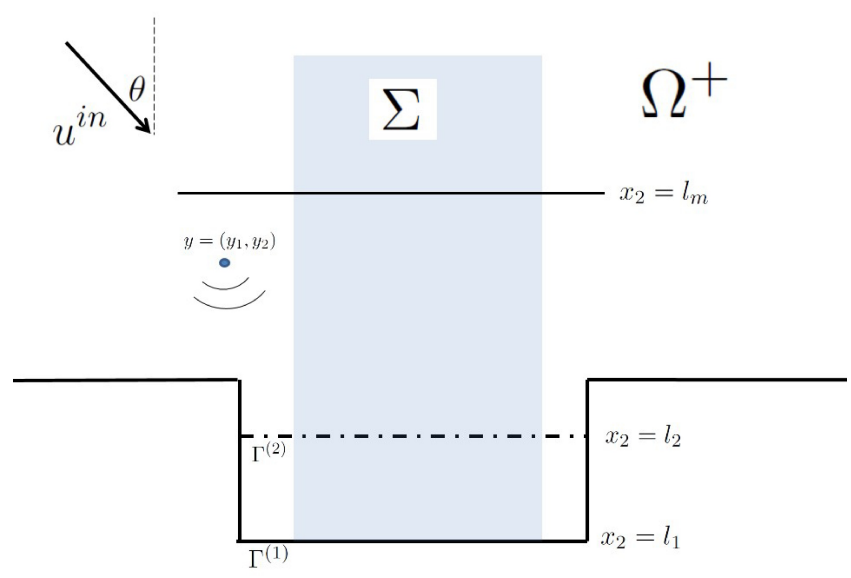

FIG. 3. Illustration of the shape of two different rectangular cavities $\Gamma^{(1)}$ and $\Gamma^{(2)}$, which generate identical outgoing waves for an incident plane or point source wave.

Since $u_{j}$ is analytic in $x_{2}>R$ and the measurement surface $I$ can be extended to an entire straight line $\Gamma_{h}:=\left\{x: x_{2}=h\right\}$ in $x_{2}>R$, we have $u_{1}=u_{2}$ on $\Gamma_{h}$. By uniqueness to the Dirichlet boundary value problem in a half-plane (see, e.g., [9]), we see $u_{1}=u_{2}$ for all $x_{2}>h$. Applying the unique continuation yields $u_{1}=u_{2}$ in $\Omega_{1}^{+} \cap \Omega_{2}^{+}$and in particular

$$
\partial_{\nu} u_{1}+i k \lambda u_{1}=\partial_{\nu} u_{2}+i k \lambda u_{2} \quad \text { on } \quad \partial\left(\Omega_{1}^{+} \cap \Omega_{2}^{+}\right) .
$$

Assume on the contrary that $\Lambda_{R}^{(1)} \neq \Lambda_{R}^{(2)}$. Since $\Lambda_{R}^{(j)}(j=1,2)$ are of rectangular type and fulfill the assumption (3.1), one can always find an infinite strip $\Sigma=(a, b) \times\left[l_{1}, \infty\right)$ which contains at least two line segments, $L_{j} \subset \Gamma^{(j)}(j=1,2)$, parallel to the $x_{1}$-axis such that $u_{1}$ (or $u_{2}$ ) satisfies the impedance boundary condition on both $L_{1}$ and $L_{2}$ and that $\Sigma \subset \Omega_{1}^{+}$(or $\Sigma \subset \Omega_{2}^{+}$). Without loss of generality, we may suppose that (see Figure 3)

$$
\Sigma \subset \Omega_{1}^{+}, \quad L_{j}=\left\{\left(x_{1}, l_{j}\right): x_{1} \in(a, b)\right\} \subset \Lambda_{R}^{(j)}, \quad l_{2}>l_{1}, \quad\left|l_{j}\right|<H .
$$

This implies that $v:=u_{1}$ is a solution to the Helmholtz equation in $\Sigma$ satisfying $\partial_{2} v+i k \lambda v=0$ on $L_{1} \cup L_{2}$. Define $w:=\partial_{2} v+i k \lambda v$. We see that $w$ still satisfies the Helmholtz equation in $\Sigma$ with the Dirichlet boundary condition on $L_{1} \cup L_{2}$. Applying reflection principle for the Helmholtz equation (see, e.g., [19]) to $w$ yields the vanishing of $w$ on an infinite number of line segments in $\Sigma$, i.e., $w=0$ on $L_{n}$ with

$$
L_{n}:=\left\{\left(x_{1}, l_{n}\right): x_{1} \in(a, b), l_{n}=l_{1}+(n-1)\left(l_{2}-l_{1}\right)\right\} \quad \text { for all } n \in \mathbb{N} .
$$

Choose $m \in \mathbb{N}$ sufficiently large such that $l_{m}>R$. This implies

$$
w:=\partial_{2} u_{1}+i k \lambda u_{1}=0 \quad \text { on } \quad x_{2}=l_{n} \text { for all } n \geq m .
$$

Below we shall consider incident plane and point source waves separately.

Case (i): $u^{i n}(x)=\exp \left(i k\left(x_{1} \sin \theta-x_{2} \cos \theta\right)\right)$ for $\theta=\theta_{j} \in(-\pi / 2, \pi / 2)(j=$ $1,2, \ldots, 2 M+1)$ are plane waves at the fixed wavenumber $k \in \mathbb{R}^{+}$. 
In this case, the total field $u_{1}$ is of the form (see Theorem 2.3)

$$
u_{1}=e^{i k\left(x_{1} \sin \theta-x_{2} \cos \theta\right)}+\frac{\cos \theta-\lambda}{\cos \theta+\lambda} e^{i k\left(x_{1} \sin \theta+x_{2} \cos \theta\right)}+u_{1}^{s c} \quad \text { in } \quad|x|>R,
$$

where $u_{1}^{s c}$ satisfies the Sommerfeld radiation condition (2.9). Inserting (3.3) into (3.2) and making straightforward calculations show that

(3.4) $A_{n} e^{i k x_{1} \sin \theta}+\partial_{2} u_{1}^{s c}\left(x_{1}, l_{n}\right)+i k \lambda u_{1}^{s c}\left(x_{1}, l_{n}\right)=0 \quad$ for all $\quad x_{1} \in \mathbb{R}, \quad n \geq m$,

where the constant $A_{n}=A_{n}(\theta, k, \lambda) \in \mathbb{C}$ is given by

$$
A_{n}=i k(\lambda-\cos \theta) e^{-i k l_{n} \cos \theta}+i k(\cos \theta-\lambda) e^{i k l_{n} \cos \theta} .
$$

Due to the decay of $u_{1}^{s c}$ when $x_{1} \rightarrow \infty$, we conclude from (3.4) that $A_{n}=0$ for all $n \geq m$, implying that

$$
\exp (2 i s k \cos \theta)=1, \quad s:=l_{2}-l_{1}>0 .
$$

Hence, $s k \cos \theta=\eta \pi$ for some $\eta \in \mathbb{Z}$. Recalling that $2 H>s>0$ and $\theta \in(-\pi / 2, \pi / 2)$, we obtain

$$
0<\eta=(s k \cos \theta) / \pi<(2 H k) / \pi .
$$

This implies that for every fixed incident angle $\theta_{j} \in(-\pi / 2, \pi / 2)$, there exists an integer $l_{j}$ such that the previous relation holds with $\eta=\eta_{j} \leq M$. Moreover, we have $\eta_{j}=\eta_{j^{\prime}}$ if and only if $\theta_{j}=\theta_{j^{\prime}}$ or $\theta_{j}=-\theta_{j^{\prime}}$. Hence, it is impossible that the relation (3.5) holds for $2 M+1$ distinct directions $\theta_{j}(j=1,2, \ldots, 2 M+1)$. This contradiction yields $\Lambda_{R}^{(1)}=\Lambda_{R}^{(2)}$.

Case (ii): $u^{i n}(x)=\exp \left(i k\left(x_{1} \sin \theta-x_{2} \cos \theta\right)\right)$ for $k=k_{j}<K(j=1,2, \ldots, N+1)$ are plane waves with fixed $\theta \in(-\pi / 2, \pi / 2)$.

As done in Case (i), we conclude that for every $k_{j}$ the relations

$$
\left(l_{2}-l_{1}\right) k_{j} \cos \theta=\eta_{j} \pi, \quad 0<\eta_{j}<2 H K / \pi,
$$

hold for some integer $\eta_{j} \leq N$. Further, we have $\eta_{j}=\eta_{j^{\prime}}$ if and only if $k_{j}=k_{j^{\prime}}$. Hence, if $u_{1}$ coincides with $u_{2}$ on $I$ for $N+1$ distinct wavenumbers, then the relation $0<\eta_{j} \leq N$ must hold for all $j=1,2, \ldots, N+1$. However, it is impossible since $\eta_{j} \neq \eta_{j^{\prime}}$. This proves $\Lambda_{R}^{(1)}=\Lambda_{R}^{(2)}$.

Case (iii): $u^{\text {in }}(x)=i / 4 H_{0}^{(1)}(k|x-y|)$ is a point source wave with fixed $k \in \mathbb{R}^{+}$ and $y \in \Omega_{R}^{+}$.

Analogously to the plane wave case, the unique solution can be written as $u_{1}(x)=$ $G(x, y)+u_{1}^{s c}(x)$ in $|x|>R$, where $G(x, y)$ is the half-space Green's function with the impedance boundary condition on $x_{2}=0$. Denote by $y^{*}$ the reflection of the point source with respect to the line $\Gamma_{\eta_{m}}$, i.e., $y^{*}=\left(y_{1}, y_{2}+2\left(\eta_{m}-y_{2}\right)\right)$ for $y_{2}<\eta_{m}$. On the one hand, we have $\left|u_{1}\left(y^{*}\right)\right|<\infty$ since $y^{*} \in \Omega_{1}$. On the other hand, using the extension formula for the Helmholtz equation across an impedance surface $\Gamma_{\eta_{m}}$, we may express $u_{1}\left(y^{*}\right)$ in terms of the values of $u_{1}(x)$ for $x \in\left\{\left(y_{1}, x_{2}\right): x_{2} \in\left(y_{2}, \eta_{m}\right)\right\}$ as follows (see, e.g., $[23,19])$ :

$$
u_{1}\left(y^{*}\right)=u_{1}(y)-2 i k \lambda \int_{y_{2}}^{l_{m}} e^{-\left(y_{2}+t\right) i k \lambda} u_{1}\left(y_{1}, t\right) d t .
$$

This implies that $u_{1}\left(y^{*}\right)=\infty$, since $u_{1}(x)=1 /(2 \pi) O(\ln (1 /|x-y|))$ as $x \rightarrow y$. This contradiction implies that $\Lambda_{R}^{(1)}=\Lambda_{R}^{(2)}$. 
Remark 3.1.

(i) It follows from Theorem 3.1 that more plane waves are needed for detecting rectangular cavities which are large and deep. In the first assertion of Theorem 3.1, the number of incident directions can be reduced to $M+1$ if the incident angles are all required to be nonpositive or nonnegative.

(ii) The proof of Theorem 3.1 relies on the reflection principle for the Helmholtz equation under the Dirichlet boundary condition. This property is applied to the function $\partial_{2} u+i \lambda u$ if the Robin coefficient $\lambda$ is known in advance. It is unclear to us how to carry out the proof if $\lambda$ is not given.

(iii) By arguing the same way as the proof of Theorem 3.1, one can prove that a bounded rectangular obstacle of impedance type can be uniquely determined by the far-field pattern of a single plane or point source wave.

4. Numerical tests. In this section, we present several numerical examples to verify the efficiency of the proposed finite element method coupled with the boundary element method in a locally perturbed half-plane. Below we shall consider acoustic wave scattering problems with Dirichlet, Neumann, or impedance boundary conditions enforced on $\Gamma:=\left\{x: x_{2}=f\left(x_{1}\right)\right\}$. For simplicity we assume that the inhomogeneous medium is absent, i.e., $D=\emptyset$ and $q \equiv 1$ in $\Omega^{+}$. The truncated domain $\Omega_{R}^{+}$is discretized by uniform triangle elements using the MATLAB toolbox pdetool and the standard piecewise linear basis functions $\left\{\varphi_{i}\right\}_{i=1}^{N}$ are employed to construct the finite element space on $\Omega_{R}^{+}$. Here $N$ is the total number of nodes in $\Omega_{R}^{+}$and we denote by $\left\{x_{j}\right\}_{j=1}^{N_{R}}$ the nodes on $S_{R}^{+}$. Then we use piecewise constant basis functions $\left\{\psi_{j}\right\}_{j=1}^{N_{R}-1}$ to construct the boundary element space, where $\psi_{j}$ is defined on the line segment $S_{R, j}^{+}$with vertices $x_{j}$ and $x_{j+1}$. In particular, the combination of $N_{R}-1$ segments $S_{R, 1}^{+}, S_{R, 2}^{+}, \ldots, S_{R, N_{R}-1}^{+}$gives an approximation of $S_{R}^{+}$. Denote

$$
e_{1}:=\left\|u^{s c}-u_{h}^{s c}\right\|_{L^{2}\left(\Omega_{R}^{+}\right)}, \quad e_{2}:=\left\|u^{s c}-u_{h}^{s c}\right\|_{H^{1}\left(\Omega_{R}^{+}\right)}, \quad e_{3}:=\left\|p-p_{h}\right\|_{L^{2}\left(S_{R}^{+}\right)} .
$$

It should be pointed out that there is no limit that the boundary integral equations for the scattered field can only be derived on $S_{R}^{+}$. In fact, the boundary integral equations (2.16) hold true for any smooth open arc whose ending points are located on $\Gamma \backslash \Lambda_{R}$.

Example 1. In this example, the local perturbation of the infinite plane is supposed to be given by the graph of the piecewise smooth function

$$
f\left(x_{1}\right)= \begin{cases}\sqrt{R_{0}^{2}-x_{1}^{2}}, & -R_{0} \leq x_{1} \leq R_{0}, \\ 0, & \text { otherwise }\end{cases}
$$

which lines above the $x_{1}$-axis. The incident field is set to be a point source $u^{i n}(x)=$ $-\frac{i}{4} H_{0}^{(1)}(k|x-z|), x \neq z$, emitting from the source position located at $z=(0,0.5)$ lying below $\Gamma$. Then the scattered and reflected fields are given, respectively, by

$$
\begin{aligned}
u^{r e} & =\frac{i}{4} H_{0}^{(1)}(k|x-z|)-\frac{i}{4} H_{0}^{(1)}\left(k\left|x-z^{\prime}\right|\right), \\
u^{s c} & =\frac{i}{4} H_{0}^{(1)}(k|x-z|)-\frac{i}{4} H_{0}^{(1)}\left(k\left|x-z^{\prime}\right|\right)
\end{aligned}
$$

in the Dirichlet case, and by

$$
\begin{aligned}
u^{r e} & =-\frac{i}{4} H_{0}^{(1)}(k|x-z|)-\frac{i}{4} H_{0}^{(1)}\left(k\left|x-z^{\prime}\right|\right), \\
u^{s c} & =\frac{i}{4} H_{0}^{(1)}(k|x-z|)+\frac{i}{4} H_{0}^{(1)}\left(k\left|x-z^{\prime}\right|\right)
\end{aligned}
$$

Copyright (c) by SIAM. Unauthorized reproduction of this article is prohibited. 
TABLE 1

Numerical errors for Example 1 with $k=1$ in the Dirichlet case.

\begin{tabular}{|c|c|c|c|c|c|c|}
\hline$h$ & $e_{1}$ & Order & $e_{2}$ & Order & $e_{3}$ & Order \\
\hline 0.4040 & $3.73 \mathrm{E}-3$ & - & $5.19 \mathrm{E}-2$ & - & $1.74 \mathrm{E}-2$ & - \\
0.2098 & $9.14 \mathrm{E}-4$ & 2.15 & $2.59 \mathrm{E}-2$ & 1.06 & $8.70 \mathrm{E}-3$ & 1.06 \\
0.1068 & $2.21 \mathrm{E}-4$ & 2.10 & $1.29 \mathrm{E}-2$ & 1.03 & $4.34 \mathrm{E}-3$ & 1.03 \\
0.0539 & $5.21 \mathrm{E}-5$ & 2.11 & $6.47 \mathrm{E}-3$ & 1.01 & $2.17 \mathrm{E}-3$ & 1.01 \\
0.0271 & $1.18 \mathrm{E}-5$ & 2.16 & $3.23 \mathrm{E}-3$ & 1.01 & $1.08 \mathrm{E}-3$ & 1.01 \\
\hline
\end{tabular}

TABLE 2

Numerical errors for Example 1 with $k=5$ in the Neumann case.

\begin{tabular}{|c|c|c|c|c|c|c|}
\hline$h$ & $e_{1}$ & Order & $e_{2}$ & Order & $e_{3}$ & Order \\
\hline 0.4040 & $5.65 \mathrm{E}-2$ & - & $4.78 \mathrm{E}-1$ & - & $4.15 \mathrm{E}-1$ & - \\
0.2098 & $1.55 \mathrm{E}-2$ & 1.97 & $2.22 \mathrm{E}-1$ & 1.17 & $1.25 \mathrm{E}-1$ & 1.83 \\
0.1068 & $3.99 \mathrm{E}-3$ & 2.02 & $1.08 \mathrm{E}-1$ & 1.07 & $4.11 \mathrm{E}-2$ & 1.65 \\
0.0539 & $1.01 \mathrm{E}-3$ & 2.01 & $5.36 \mathrm{E}-2$ & 1.02 & $1.65 \mathrm{E}-2$ & 1.33 \\
0.0271 & $2.53 \mathrm{E}-4$ & 2.01 & $2.67 \mathrm{E}-2$ & 1.01 & $7.66 \mathrm{E}-3$ & 1.12 \\
\hline
\end{tabular}

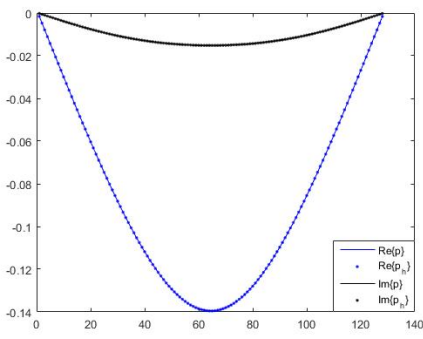

(a) $k=1$

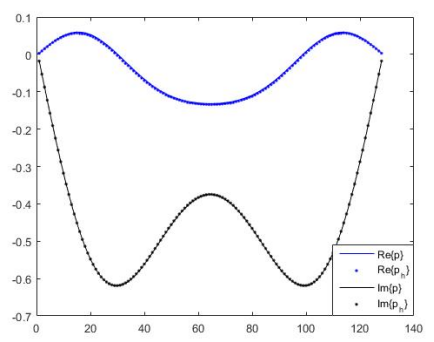

(b) $k=5$

FIG. 4. The exact and numerical solutions of $p$ for Example 1 with $h=0.0539$ in the Dirichlet case.

in the Neumann case, where $z^{\prime}=(0,-0.5)$. These analytical formulas can be used to examine the validity of our approach. Tables 1 and 2 list the $L^{2}$ and $H^{1}$ errors of $u^{s c}$ in $\Omega_{R}^{+}$and the $L^{2}$ error of $p=\partial_{\nu} u^{s c}$ on $S_{R}^{+}$, respectively. The convergence rate as a function of the finite element mesh size $h$ (see also Tables 1 and 2) shows the convergence order of $O\left(h^{2}\right)$ in the $L^{2}\left(\Omega_{R}^{+}\right)$norm and the order of $O(h)$ in the both $H^{1}\left(\Omega_{R}^{+}\right)$norm and $L^{2}\left(S_{R}^{+}\right)$norm. In Figure 4 , we plot the real and imaginary parts of the numerical solution $p_{h}$. They are in a perfect agreement with the exact ones from both quantitative and qualitative points of view.

Example 2. In this example, we first consider the scattering of plane incident wave if $\Omega^{-}$is of impendence type in the absence of local perturbation and check whether our code provides the correct solution. Then the total field is given by (cf. (2.5))

$$
\begin{aligned}
u=u^{i n}+u^{r e}= & \exp \left(i k\left(x_{1} \sin \theta-x_{2} \cos \theta\right)\right) \\
& +\frac{\cos \theta-\lambda}{\cos \theta+\lambda} \exp \left(i k\left(x_{1} \sin \theta+x_{2} \cos \theta\right)\right), \quad \theta \in(-\pi / 2, \pi / 2) .
\end{aligned}
$$

Choose $k=3, \lambda=1$, and $\theta=-\pi / 3$. Table 3 presents the $L^{2}\left(\Omega_{R}^{+}\right)$and $H^{1}\left(\Omega_{R}^{+}\right)$errors of $u$ and their convergence rates as functions of the finite element mesh size $h$. In Figure 5, we show the real part of the exact and numerical solutions of the total field, which are in a perfect agreement with each other from a qualitative point of view. 
TABLE 3

Numerical errors for Example 2.

\begin{tabular}{|c|c|c|c|c|}
\hline$h$ & $e_{1}$ & Order & $e_{2}$ & Order \\
\hline 0.4630 & $4.68 \mathrm{E}-1$ & - & $2.47 \mathrm{E} 0$ & - \\
0.2315 & $1.38 \mathrm{E}-1$ & 1.76 & $1.16 \mathrm{E} 0$ & 1.09 \\
0.1173 & $3.64 \mathrm{E}-2$ & 1.96 & $5.64 \mathrm{E}-1$ & 1.06 \\
0.0592 & $9.23 \mathrm{E}-3$ & 2.01 & $2.79 \mathrm{E}-1$ & 1.03 \\
\hline
\end{tabular}

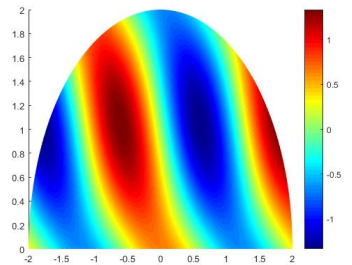

(a) $\operatorname{Re} u$

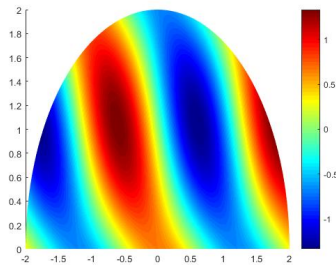

(b) $\operatorname{Re} u_{h}$

FIG. 5. The exact and numerical solutions of the total field for Example 2.

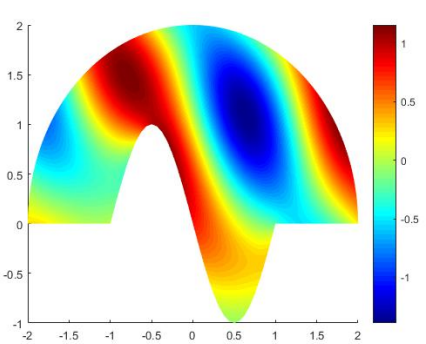

(a) $\operatorname{Re} u_{h}$

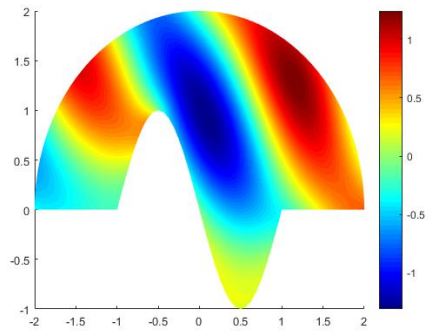

(b) $\operatorname{Im} u_{h}$

FIG. 6. The numerical solution of the total field for Example 2 where the local perturbation is sin-type.

Finally, we consider the case of a general scattering interface with an impendence boundary condition. The local perturbation of the infinite plane is given by

$$
f\left(x_{1}\right)= \begin{cases}-\sin \left(\pi x_{1} / R_{0}\right), & -R_{0} \leq x_{1} \leq R_{0}, \\ 0 & \text { otherwise }\end{cases}
$$

Figure 6 presents the numerical results using the finite element method coupled with the boundary element method.

Acknowledgment. We thank the referees for their constructive comments which helped to improve the paper.

\section{REFERENCES}

[1] H. Ammari, G. Bao, And A. Wood, An integral equation method for the electromagnetic scattering from cavities, Math. Methods Appl. Sci., 23 (2000), pp. 1057-1072.

[2] H. Ammari, G. BaO, AND A. Wood, Analysis of the electromagnetic scattering from a cavity, Jpn. J. Ind. Appl. Math., 19 (2002), pp. 301-310.

[3] G. BAO, J. GaO, AND P. LI, Analysis of direct and inverse cavity scattering problems, Numer. Math. Theor. Meth. Appl., 4 (2011), pp. 419-442. 
[4] G. BAO AND J. Lin, Imaging of local surface displacement on an infinite ground plane: The multiple frequency case, SIAM J. Appl. Math., 71 (2011), pp. 1733-1752.

[5] G. BAO AND J. Lin, Imaging of reflective surfaces by near-field optics, Opt. Lett., 37 (2012), pp. 5027-5029.

[6] G. BAO AND J. Lin, Near-field imaging of the surface displacement on an infinite ground plane, Inverse Probl. Imaging, 2 (2013), pp. 377-396.

[7] S. N. Chandler-Wilde, Ground Effects in Environmental Sound Propagation, Ph.D theses, University of Bradford, UK, 1988.

[8] S. N. Chandler-Wilde, The impedance boundary value problem for the Helmholtz equation in a half-plane, Math. Methods Appl. Sci., 20 (1997), pp. 813-840.

[9] S. N. Chandler-Wilde And J. Elschner, Variational approach in weighted Sobolev spaces to scattering by unbounded rough surfaces, SIAM J. Math. Anal., 42 (2010), pp. 2554-2580.

[10] S. N. Chandler-Wilde And D. C. Hothersall, Efficient calculation of the green function for acoustic propagation above a homogeneous impedance plane, J. Sound Vib., 180 (1995), pp. 705-724.

[11] S. N. Chandler-Wilde AND P. Monk, Existence, uniqueness and variational methods for scattering by unbounded rough surfaces, SIAM J. Math. Anal., 37 (2005), pp. 598-618.

[12] S. N. Chandler-Wilde And A. T. Peplow, A boundary integral equation formulation for the Helmholtz equation in a locally perturbed half-plane, ZAMM Z. Angew. Math. Mech., 85 (2005), pp. 79-88.

[13] S. N. Chandler-Wilde And B. Zhang, Electromagnetic scattering by an inhomogeneous conducting or dielectric layer on a perfectly conducting plate, Proc. Roy. Soc. London A, 454 (1998), pp. 519-542.

[14] S. N. Chandler-Wilde And B. Zhang, A uniqueness result for scattering by infinite rough surfaces, SIAM J. Appl. Math., 58 (1998), pp. 1774-1790.

[15] L. Chorfi And P. Gaitan, Reconstruction of the interface between two-layered media using far-field measurements, Inverse Problems, 27 (2011), 075001.

[16] D. Colton And R. Kress, Inverse Time-Harmonic Acoustic and Electromagnetic Scattering Theory, Springer-Verlag, New York, 2013.

[17] D. Colton and B. D. Sleeman, Uniqueness theorems for the inverse problem of acoustic scattering, IMA J. Appl. Math., 31 (1983), pp. 253-259.

[18] J. A. DeSAnto, Scalar Wave Theory: Green's Functions and Applications, Springer Ser. Wave Phenomena 12, Springer-Verlag, Berlin, 1992.

[19] J. B. Diaz AND G. S. LudFord, Reflection principles for linear elliptic second order partial differential equations with constant coefficients, Ann. Mat. Pura Appl., 39 (1955), pp. 87-95.

[20] J. ElsChNER AND G. Hu, Elastic scattering by unbounded rough surfaces: Solvability in weighted sobolev spaces, Appl. Anal., 94 (2015), pp. 251-278.

[21] G. C. Hsiao and W. L. Wendland, Boundary Integral Equations, Springer-Verlag, Berlin, 2008.

[22] G. HU, X. LIU, F. QU, AND B. ZHANG, Variational approach to rough surface scattering problems with Neumann and generalized impedance boundary conditions, Commun. Math. Sci., 13 (2015), pp. 511-537.

[23] G. Hu And M. Yамамото, Hölder stability estimate of the robin coefficient in corrosion detection problems with a single boundary measurement, Inverse Problems, 31 (2015), 115009.

[24] J. M. JIN, Electromagnetic scattering from large, deep, and arbitrarily-shaped open cavities, Electromagnetics, 18 (1998), pp. 3-34.

[25] G. Kristensson, Uniqueness theorems for the Helmholtz equation: Penetrable media with an infinite interface, SIAM J. Math. Anal., 11 (1980), pp. 1104-1116.

[26] P. LI, Coupling of finite element and boundary integral methods for electromagnetic scattering in a two-layered medium, J. Comput. Phys., 229 (2010), pp. 481-497.

[27] P. LI, A survey of open cavity scattering problems, J. Comp. Math., 36 (2018), pp. 1-16.

[28] W. MClean, Strongly Elliptic Systems and Boundary Integral Equations, Cambridge University Press, Cambridge, 2000.

[29] F. M. ODeH, Uniqueness theorems for the Helmholtz equation in domains with infinite boundaries, J. Math. Mech., 12 (1963), pp. 857-868.

[30] G. F. Roach And B. Zhang, On Sommerfeld radiation conditions for the diffraction problem with two unbounded media, Proc. Roy. Soc. Edinburgh Sect. A, 121 (1992), pp. 149-161.

[31] G. F. ROACH AND B. ZHANG, A transmission problem for the reduced wave equation in inhomogeneous media with an infinite interface, Proc. Roy. Soc. London Ser. A, 436 (1992), pp. $121-140$.

Copyright (c) by SIAM. Unauthorized reproduction of this article is prohibited. 
[32] A. Willers, The Helmholtz equation in disturbed half-spaces, Math. Methods Appl. Sci., 9 (1987), pp. 312-323.

[33] A. WoOD, Analysis of electromagnetic scattering from an overfilled cavity in the ground plane, J. Comput. Phys., 215 (2006), pp. 630-641.

[34] B. Zhang, On transmission problems for wave propagation in two locally perturbed half-spaces, Math. Proc. Cambridge Philos. Soc., 115 (1994), pp. 545-558.

[35] B. Zhang and S. N. Chandler-Wilde, Integral equation methods for scattering by infinite rough surfaces, Math. Methods Appl. Sci., 26 (2003), pp. 463-488.

[36] H. Zhang AND B. ZhANG, A novel integral equation for scattering by locally rough surface and applications to the inverse problem, SIAM J. Appl. Math., 73 (2013), pp. 1811-1829.

Copyright (c) by SIAM. Unauthorized reproduction of this article is prohibited. 